\title{
Diagnosing of rice nitrogen stress based on static scanning technology and image information extraction
}

\author{
L.S. Chen ${ }^{1,2}$, K. Wang ${ }^{1,2 *}$ \\ ${ }^{1}$ Institute of Applied Remote Sensing \& Information Technology, Zhejiang University, Hangzhou, 310058 China. ${ }^{2}$ Ministry of \\ Education Key Laboratory of Environmental Remediation and Ecological Health, Zhejiang University, Hangzhou 310058, China. \\ *Corresponding author: kwang@zju.edu.cn
}

\begin{abstract}
At present, the identifying of rice nitrogen stress by the chemical analysis is time-consuming and laborious. Machine vision technology can be used to non-destructively and rapidly identify rice nitrogen status, but image acquisition via digital camera is vulnerable to external conditions, and the images are of poor quality. In this research static scanning technology was used to collect images of the rice's top-three leaves that were fully expand in 4 growth periods. From those images, 14 spectral and shape characteristic parameters were extracted by R, G, B mean value function and Regionprops function in MATLAB. After analyzing, the R, G, Leaf Length, Leaf Area, and Leaf Perimeter were chosen as 5 universal characteristic parameters for identifying nitrogen stress in 4 growth periods.
\end{abstract}

The results showed that the overall recognition accuracy of nitrogen stress were $92 \%, 92 \%, 100 \%$ and $96 \%$ respectively. Based on the result, the methodology developed in the study is capable of identifying nitrogen stress accurately in the rice.

Keywords: Static scanning, rice, nitrogen stress, SVM, LOO-CV

\section{Introduction}

Nitrogen $(\mathrm{N})$ is one of the indispensable minerals for plant growth. It is particularly important for promoting root development, flowering and ripening. However, most of the world's agricultural soils are deficient in $\mathrm{N}$ supply. Using mineral $\mathrm{N}$ fertilizer can effectively overcome this problem. While the excessive use of $\mathrm{N}$ fertilizer may increase the risk of environmental pollution and increase production cost. Therefore, quick and accurate diagnosis of rice $\mathrm{N}$ nutrient status is necessary for guiding $\mathrm{N}$ fertilizer.

Rice with nitrogen deficiency usually has no significant symptoms before tillering stage. After the tillering stage its growth slows down significantly. Nitrogen deficiency most often results in stunted growth, slow growth, and chlorosis with small unit leaf area. 
Because nitrogen is mobile, the older leaves exhibit chlorosis and necrosis earlier than the younger leaves. Light green to yellow appearance of leaves, especially older leaves. Leaf color can indicate the plant nutrient and health status which is closely related to the amount and proportion of nitrogen content. Plant leaf color analysis has proved to be useful in assessing plant nitrogen level, plant in image analysis. Therefore, this study used scanning as the acquisition method. Since scanning is in a closed environment, which can reduce the influence of external conditions, and ensures the reproducibility of the color and other characteristics. This method is seldom used for assessing crop nutrition.

At present, common methods for crop assessment include Bayesian classification, decision tree, neural network, etc. For example, recognition of rice disease based by Bayes method (Z. X. Guan et al., 2010), study on discrimination of corn seed based on nearInfrared spectra and artificial neural network model (J. Chen et al., 2008), disease, and senescence.

In general, the diagnosis of nitrogen deficiency is based on chemical analysis in the laboratory. But such methods are destructive, time-consuming, and difficult to apply in the field. However, there is a correlation between the chemical composition of leaf tissues and reflectance in the visible spectrum (J.W. Lu et al., 2010), digital color image analysis might be capable of early diagnosis of nitrogen stress (L. Bacci et al., 1998). Previous studies had used digital camera (L. L. Jia et al., 2009) and aerial Photography (Blackmer et al., 1996) to directly acquire images for diagnosis of crop nutrient status. With simple and convenient operation the digital camera can obtain images, but the image acquisition process is affected by external conditions, and the acquired images usually have complex background, multi-redundant information and image noise, which may cause problem application of Clustering based decision Tree in the screening of maize germplasm (B. Wang et al., 2011) have been used. Those methods are suitable for the larger sample size, but have low recognition rate for the small sample size. Currently, on the basis of Vapnik-Chervonenkis dimension theory and structural risk minimization principle of statistics, an all-purpose machine learning algorithm is created by Vapnik: support vector machine (SVM) that is suitable for small sample classification (N.Y. Deng et al., 2004; G.Z. Li et al., 2004). The advantage of SVM than earlier methods is that SVM is usually less vulnerable to over fitting problem (H. Wei et al., 2005), because SVM is designed to minimize structural risk, and previous techniques are usually based on minimizing empirical risk. Because of those advantages SVM has been used in studies such as land cover change detection (H. Nemmour et al., 2006), modeling spectral mixtures (L. Brown et al., 2000), Pattern recognition and data mining (Christopher et al., 1998), soil type classification (Milos Kovacevica et al., 2010), identification diagnosis system of rice insect pests (J.J. Shi et al., 2009). However, less attention has been paid to the identification of rice nutrition stress.

In this study, the scanning images of rice leaves under different level of nitrogen nutrition were selected as the research objective to identify the nitrogen stress using 5 universal characteristic parameters in spectral and shape (R, G, Leaf Length, Leaf Area, and Leaf Perimeter). This study can provide the theoretical evidence for the diagnosis of rice nitrogen deficiency and guiding fertilization in time.

\section{Materials and Methods}

\subsection{Experimental design}

The experiment was designed to study the rice of different $\mathrm{N}$ status. Seeds of rice (cultivar ZheYouNO.1) were pre-germinated during 3 days, in moist sand at $30{ }^{\circ} \mathrm{C}$ and seedlings were individually transplanted 7 days after emergence in $20 \mathrm{~L}$ polyvinyl chloride (PVC) pots which contained clean, sieved, and thoroughly leached river sand allowing precise control of nutrient. The experiment was carried out in a glasshouse at ZiJinGang campus, Zhejiang University 
$\left(30^{\circ} 17^{\prime} \mathrm{N}, 120^{\circ} 05^{\prime} \mathrm{E}\right.$, Hangzhou, China). Plants were grown under natural light conditions. The temperature of the glasshouse was programmed at $30{ }^{\circ} \mathrm{C} / 25{ }^{\circ} \mathrm{C}$ (day/night) and the relative humidity at $50 \%$. The nutrient solution was prepared with deionized water and contained $\mathrm{NaH}_{2} \mathrm{PO}_{4} \cdot 2 \mathrm{H}_{2} \mathrm{O} 10.66 \mathrm{mg} / \mathrm{L}, \mathrm{K}_{2} \mathrm{SO}_{4}$ $33.6 \mathrm{mg} / \mathrm{L}, \mathrm{CaCl}_{2} 32.94 \mathrm{mg} / \mathrm{L}, \mathrm{MgSO}_{4} .7 \mathrm{H}_{2} \mathrm{O} 54.32$ $\mathrm{mg} / \mathrm{L}, \mathrm{Na}_{2} \mathrm{SiO}_{3} .9 \mathrm{H}_{2} \mathrm{O} 16.5 \mathrm{mg} / \mathrm{L}$. The experiment had five different $\mathrm{N}$ level treatments, 5 replications for each level, and 5 rice plants in each pot. Five $\mathrm{N}$ levels (ammonium nitrate $0 \mathrm{mg} / \mathrm{L}, 6 \mathrm{mg} / \mathrm{L}, 12 \mathrm{mg} / \mathrm{L}, 18 \mathrm{mg} / \mathrm{L}$ and $24 \mathrm{mg} / \mathrm{L}$, respectively.) via hydroponic solutions were applied to different pots. The nutrient solutions in pots were replaced every 14 days. Every 5 days the $\mathrm{pH}$ of the nutrient solution in each pot were measured and adjusted to $5.5-6.5$ using $1 \mathrm{~mol} / \mathrm{L} \mathrm{NaOH}$.

\subsection{Acquisition and analysis images of rice leaves}

Leaf samples were taken on the August $4^{\text {th }}$, August $18^{\text {th }}$, August $27^{\text {th }}$, and September $8^{\text {th }}$ in 2011. The top-three leaves (L.G. Jiang et al., 2004; J.X. Zhu et al., 2009) in 4 rice plants of 5 nitrogen levels were collected at each sampling time.
After 4 dates were completed, a total of 300 rice leaves were processed with the following manner at the same day as they were collected, which were operated in the laboratory.

First, leaves were placed on a scanner (EPSON GT20000), scanner with a maximum scanning area of $11.7 \times 17.0$ inches, a R/G/B (The full color images consist of red (R), green $(G)$, and blue (B) channels) and $\mathrm{BK}$ color $\mathrm{CCD}$ line sensor, output image data was 16 bits per pixel per color internal and 1 to 8 bits per pixel per color extern. Resolution was set to $300 \mathrm{dpi}$ (dots per inch). Leaf area $\left(\mathrm{cm}^{2}\right)$ can be calculated by the sum of all pixels within the range of leaf multiply by $(2.54 / 300)^{2}$, length $(\mathrm{cm})$ equals to the number of pixels in vein multiply by $2.54 / 300$, and width $(\mathrm{cm})$ equals to the number of pixels in the widest zone multiply by $2.54 / 300$. Through $R, G, B$ mean value function and Regionprops function in MATLAB (MathWorks Inc., USA), determining the leaf color characters $(\mathrm{R}, \mathrm{G}, \mathrm{B})$ and shape characters (leaf length, leaf width, leaf area, leaf perimeter, $\mathrm{AL}$ ratio, $\mathrm{AP}$ ratio, eccentricity, rectangularity, area convexity, circularity, form Factor) in Table 1

Table 1. The Formula and explanation of different characteristics

\begin{tabular}{|c|c|c|}
\hline Characteristics & Formula & Explanation \\
\hline AL_ratio & $A L_{-}$ratio $=\frac{\text { Area }}{\text { Length }}$ & The ratio of leaf area and leaf length \\
\hline AP_ratio & $A P_{-}$ratio $=\frac{\text { Area }}{\text { Peremeter }}$ & The ratio of leaf area and leaf perimeter \\
\hline Eccentricity & Eccentricity $=\frac{\text { AxisLength }_{\text {long }}}{\text { AxisLength }_{\text {short }}}$ & The ratio of leaf length and leaf width \\
\hline Rectangularity & Rectan gularity $=\frac{\text { Area }_{\text {object }}}{\text { Area }_{\text {bounding-box }}}$ & $\begin{array}{l}\text { The ratio of leaf area and area of the } \\
\text { smallest encasing box area of leaf }\end{array}$ \\
\hline Area Convexity & Area_convexity $=\frac{\text { Area }}{\text { Convex Area }}$ & $\begin{array}{c}\text { The ratio leaf area and area of convex } \\
\text { Hullof leaf }\end{array}$ \\
\hline Circularity & Circularity $=\frac{R_{\text {inscribedaircle }}}{R_{\text {excircle }}}$ & $\begin{array}{c}\text { The ratio of radius of inscribed circle and } \\
\text { circumcircle }\end{array}$ \\
\hline Form Factor & $S=\frac{P}{4 \cdot \sqrt{A}}$ & $\mathrm{P}, \mathrm{A}$ are the perimeter and area of leaf \\
\hline
\end{tabular}




\subsection{Research method}

\subsubsection{The selection of optimal characteristic parameters}

In this study, one-way ANOVA was used to find the optimal characteristic parameters with larger intergroup differences for modeling. One-way ANOVA is used in significance test of mean difference between two or more samples sets. A complex object usually has many factors of mutual restriction and dependence. The purpose of the Analysis of Variance is to find significant factors, the optimum level of significant factors, as well as the interactions between various significant factors by data analysis. This method divides the difference of the factor (W) between different groups into two parts, one is intragroup difference, originating from the dispersion of all samples within this group, and the other is inter-group difference, being caused by the distinction among the different groups. If the ratio (F) of intra-group and inter-group difference is near to $1, \mathrm{~W}$ has small effect for grouping, and compared to the randomness of data, the effect can be ignored. In other words, $\mathrm{W}$ is not a significant factor for grouping. Otherwise, with the bigger $\mathrm{F}$, W has the more effect for grouping.

\subsubsection{Identification of rice nitrogen stress based on SVM}

The SVM classification has two steps, training and classification. Regard the feature vectors reflecting the different categories as an input, and use an appropriate kernel function, and introduce a non-negative Relaxation term and penalty coefficient $\mathrm{C}$ to map the feature vectors reflecting the different categories from the low dimensional nonlinear space to high dimensional linear separable space. Then searching for optimal the separating hyperplane by solving linear equations in mapping space, to form the classifier. Above is the training process. Inputting the unknown category data which has already been preprocessed into classifier to classify, we can get the classification results. This research used SVM in Lib-SVM platform, and adopted RBF kernel function.

$$
K\left(X, X_{i}\right)=\exp \left\{-\frac{\left\|X-X_{i}\right\|^{2}}{2 \sigma^{2}}\right\}
$$

The classification function is

$$
f(x)=\operatorname{sgn}\left[\sum_{i=1}^{\prime} \alpha_{i} y_{i} K\left(X, X_{i}\right)+b\right]
$$

Here $\sigma$ and $\mathrm{c}$ are both kernel parameters which are calculated by grib.py of Lib-SVM

For validating the model, because the small sample size, we chose Leave-One-Out Cross Validation (LOO-CV). This method supposes $\mathrm{N}$ samples of original data divide into $\mathrm{N}$ groups. Each sample as an individual validation set, while the left $\mathrm{N}-1$ samples as the training set, then $\mathrm{N}$ models can be obtained. The average classification accuracy from those $\mathrm{N}$ models is the performance index of classifier (Wu Liang 2011). LOO-CV has two apparent advantages.

In each round, all the samples are used in training model, so it's closest to the primary distribution of samples. The result is quite reliable.

There is no stochastic factor to influence the experimental data, ensuring a reproducible experiment process.

2.3.3. Identification of rice nitrogen stress based on fisher discriminate analysis

Fisher discriminate analysis can judge which class the research object belongs to by observing and measuring variable value. Discriminate analysis establishes discriminate function by filtering the variables including more information from those variables that can show the classification and characteristic of observation object. With this method, misjudge rate can be the lowest. And its common formation is

$y=a_{1} x_{1}+a_{2} X_{2}+a_{3} x_{3}+\cdots+a_{n} X_{n}$ 
Here $\mathrm{y}$ is discriminate value, $\mathrm{x} 1, \mathrm{x} 2, \mathrm{x} 3 \ldots, \mathrm{xn}$ are variables reflecting the characters of research object, and $\mathrm{a} 1, \mathrm{a} 2, \mathrm{a} 3 \ldots, \mathrm{a}_{\mathrm{n}}$ are discriminate coefficients.

\section{Results and Discussion}

\subsection{Optimal selections of leaves' characteristic parameters in different growth period}

Samples collected from every growth period are divided into 3 types according to the P level: extreme deficiency $\left(0 \mathrm{mg} . \mathrm{L}^{-1}\right)$, medium deficiency $(6 \mathrm{mg} . \mathrm{L}-$ 1, $12 \mathrm{mg} . \mathrm{L}^{-1}$, and $\left.18 \mathrm{mg} . \mathrm{L}^{-1}\right)$ and normal (24 mg.L1), which will be represented by N1, N2 and N3, respectively. In Table 2 and 3, with the same leafposition (leaf-position ratio) of different growth period the characteristic parameter's $\mathrm{F}$ value is different, which means with different $\mathrm{N}$ levels the inter-group difference of rice leaf's characteristic parameter are different. In different leaf-position (leaf-position ratio) of the same growth period, the inter-group differences also are different. So, in different growth period, to build models and identify different degrees of rice nitrogen stress, we should choose optimal characteristic parameters according to different intergroup difference.

Figure 1 shows that the color of rice leaves in different $\mathrm{N}$ nutrient conditions were markedly different. In 4 growth stages, under nitrogen deficiency the rice leaves appeared yellow and had smaller unit leaf area, the chlorosis of rice leaves were more and more obviously from normal to extreme. With the increase of growth time, under the same nitrogen condition the etiolated area of rice leaf also showed increasing trend.

So the characteristic parameters which have biggest differences among $3 \mathrm{~N}$ nutrient levels were selected from the acquired 14 characteristic parameters to construct models for identifying rice nitrogen stress.

In addition, in order to enlarge the differences of characteristic parameter between various $\mathrm{N}$ nutrient levels, we introduce characteristic parameter ratio between the different leaf-positions (the first leaf/the third leaf, the second leaf/the third leaf, the first leaf /the second leaf).

After calculation it was found that $\mathrm{F}$ value was over 3.33 or Sig. value was less than 0.05 , characteristic parameter of rice with different level's nitrogen stress had the obvious difference. So we choose characteristic parameter whose $\mathrm{F}$ value was over 3.33 or Sig. value was less than 0.05 (Table 2 and 3 ) for identifying utilizing SVM and Fisher discriminate analysis.

\subsection{SVM modeling and validation}

In order to know the identification accuracy of the different degrees of nitrogen stress in rice's different growth period, as well as to choose the optimal leaf-position. In every growth period we chose characteristic parameters of different leaf-position (leaf-position ratio) with a less-than-0.05 Sig. value for modeling and classification, and then validating through LOO-CV.

From Figure 2, we can obtain the training accuracy of identifying different degrees of nitrogen stress with SVM, and the validation accuracy of identifying with LOO-CV. In Figure 2, On August $4^{\text {th }}$, when using the 3rd leaf's characteristic parameters to classify, the training and validation accuracy get to the highest, respectively $92 \%$ and $88 \%$. On August $18^{\text {th }}$, with $1^{\text {st }}, 2^{\text {nd }}$ and $3^{\text {rd }}$ leaf's characteristic parameters the identifying accuracy all was maximizing (92\%), and using $2^{\text {nd }}$ leaf had higher validation accuracy $(88 \%)$ than $1^{\text {st }}$ and $3^{\text {rd }}$, so this study thinks using $2^{\text {nd }}$ leaf's characteristic parameters for identifying rice nitrogen stress on August $18^{\text {th }}$ can get the best recognition accuracy and reliable result. on August $27^{\text {th }}$, choosing $1^{\text {st }}$ leaf's characteristic parameters to identify, the maximum identifying accuracy $(96 \%)$ and validation accuracy $(96 \%)$ was obtained. On August $27^{\text {th }}$ September $8^{\text {th }}$, when using the $1^{\text {st }}$ leaf's characteristic parameters $\mathrm{j}$ 


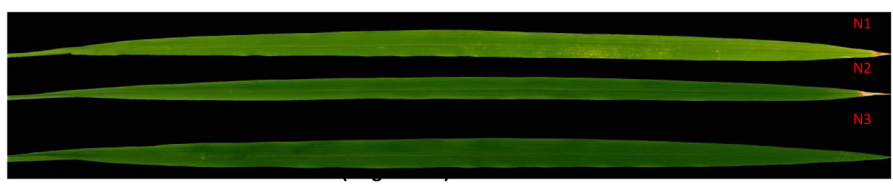

N
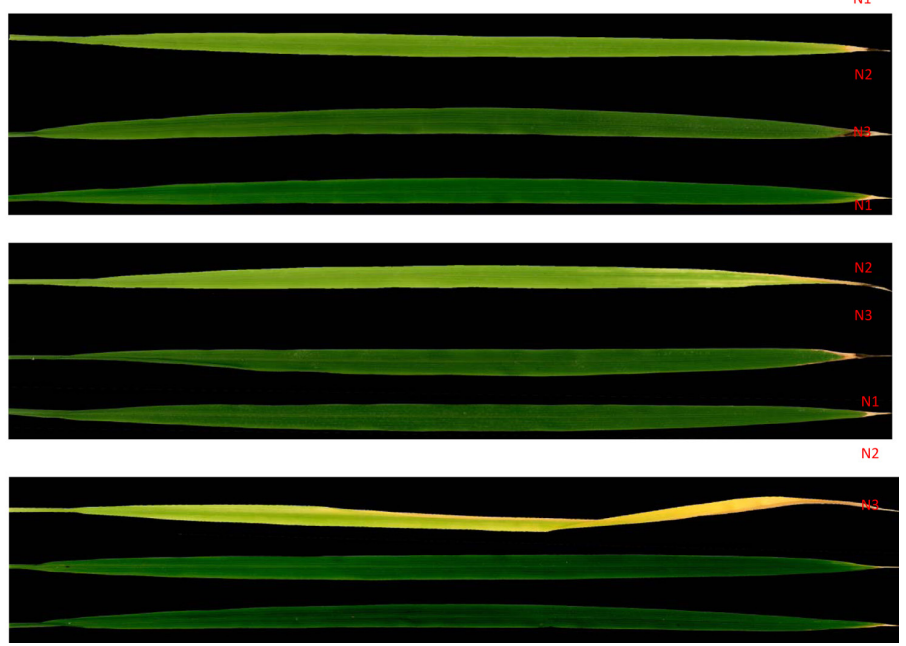

Figure 1. The difference of symptomatic characteristics in different $\mathrm{N}$ treatments ( $\mathrm{N} 1$ is extreme nutrition deficiency, $\mathrm{N} 2$ is medium nutrition deficiency, and N3 is normal nutrition level)

Table 2. F value of rice leaves' characteristic parameters by ANOVA

\begin{tabular}{|c|c|c|c|c|c|c|c|c|c|c|c|c|}
\hline & \multicolumn{4}{|c|}{ The first leaf } & \multicolumn{4}{|c|}{ The second leaf } & \multicolumn{4}{|c|}{ The third leaf } \\
\hline & 804 & 818 & 827 & 908 & 804 & 818 & 827 & 908 & 804 & 818 & 827 & 908 \\
\hline $\mathrm{R}$ & $16.38^{* * *}$ & $99.57^{* * *}$ & $63.50^{* *}$ & 2.18 & $20.64^{6 * *}$ & $185.33^{* * *}$ & $85.84^{* * *}$ & $20.39^{* * *}$ & $14.20^{* * *}$ & $78.50^{* * *}$ & $68.05^{4 * *}$ & $132.40^{* * *}$ \\
\hline G & $19.37^{* * * *}$ & $104.47^{* * * *}$ & $65.54^{x * x}$ & $4.39^{\prime \prime}$ & $20.48^{* * * *}$ & $229.89^{x * *}$ & $83.47^{* * * *}$ & $26.49^{* * * *}$ & $17.24^{* * *}$ & $123.83^{* * * *}$ & $78.53^{* * *}$ & $92.69^{* * *}$ \\
\hline B & 1.41 & 2.69 & $45.83^{* \pm *}$ & 2.59 & $3.91^{*}$ & 1.87 & $46.86^{* * *}$ & $4.04^{*}$ & $3.44^{*}$ & $7.03^{* *}$ & $49.47^{* * *}$ & $17.33^{* * *}$ \\
\hline Leaf Length & $12.15^{* * *}$ & $264.57^{* * *}$ & $53.13^{* * *}$ & $6.19^{* *}$ & $10.55^{* * *}$ & $124.28^{* * *}$ & $37.04^{* * *}$ & $14.94^{* * *}$ & $8.98^{* * *}$ & $38.86^{* * *}$ & $16.81^{* * *}$ & $16.87^{* * *}$ \\
\hline Leaf Width & 3.00 & 0.55 & 3.36 & 0.45 & $6.43^{* *}$ & 1.81 & 0.09 & 1.71 & $7.48^{* *}$ & $12.08^{* * *}$ & $20.18^{* * *}$ & 0.00 \\
\hline Leaf Area & $6.87^{* *}$ & $5.08^{\circ}$ & $9.96^{* * *}$ & $3.82^{*}$ & $11.77^{* * *}$ & $8.92^{* * *}$ & 1.81 & 0.20 & $6.94^{* *}$ & $14.74^{* * *}$ & $20.44^{* * *}$ & 2.32 \\
\hline Leaf Perimeter & $14.35^{* * *}$ & $190.77^{* * *}$ & $52.74^{* * *}$ & $6.45^{* *}$ & $16.39^{* * *}$ & $134.11^{* * *}$ & $44.81^{* * *}$ & $12.01^{* * *+}$ & $10.07^{* * *}$ & $35.79^{* * *}$ & $17.99^{* * * *}$ & $15.34^{* * *}$ \\
\hline AL_ratio & 3.00 & 0.55 & 3.36 & 0.45 & $6.43^{* *}$ & 1.81 & 0.09 & 1.71 & $7.48^{* *}$ & $12.08^{*+4}$ & $20.18^{*+*}$ & 0.00 \\
\hline AP_ratio & 3.42 & 0.82 & $3.82^{*}$ & 0.76 & $7.42^{* *}$ & 2.33 & 0.12 & 1.24 & $6.83^{* *}$ & $12.47^{*+*+}$ & $20.10^{*+* 4}$ & 0.07 \\
\hline Eccentricity & 0.42 & 2.41 & $3.98^{\circ}$ & 1.31 & 1.71 & 1.46 & 2.73 & $6.18^{* *}$ & $9.16^{* * *}$ & $8.94^{* * * *}$ & $14.05^{4 * 4}$ & $3.87^{\circ}$ \\
\hline Rectangularity & 2.45 & 2.20 & 0.14 & $4.69^{*}$ & 0.56 & $3.86^{\circ}$ & 0.61 & 0.20 & 0.80 & 1.05 & $9.02^{* * *}$ & 0.52 \\
\hline Area Convexity & 2.45 & 2.20 & 0.14 & $4.69^{*}$ & 0.56 & $3.86^{\circ}$ & 0.61 & 0.20 & 0.80 & 1.05 & $9.02^{* * *}$ & 0.36 \\
\hline Circularity & 0.33 & 3.29 & $4.05^{*}$ & 1.19 & 1.64 & 2.02 & 3.38 & $8.16^{* *}$ & $8.28^{* *}$ & $7.56^{k *}$ & $11.86^{* * *}$ & $3.96^{*}$ \\
\hline Form Factor & 0.33 & $3.60^{*}$ & $4.32^{*}$ & 1.00 & 1.58 & 2.22 & $3.92^{*}$ & $8.71^{* *}$ & $7.60^{* *}$ & $7.21^{* *}$ & $10.97^{* * *}$ & $4.00^{*}$ \\
\hline
\end{tabular}

$804,818,827$ and 908 indicate 4 growth periods (August $4^{\text {th }}$, August $18^{\text {th }}$, August $27^{\text {th }}$, and September $8^{\text {th }}$ ), respectively. The signal $\left.{ }^{*}\right)$ is the symbol of Sig. value. ${ }^{*} p<0.05 ; * * p<0.01 ; * * * p<0.001$. 
Table 3. F value of rice leaves' characteristic parameter ratio by ANOVA

\begin{tabular}{ccccccccccccc}
\hline & \multicolumn{3}{c}{ first leaf/third leaf } & \multicolumn{4}{c}{ second leaf/third leaf } & \multicolumn{4}{c}{ first leaf /second leaf } \\
\cline { 2 - 14 } & 804 & 818 & 827 & 908 & 804 & 818 & 827 & 908 & 804 & 818 & 827 & 908 \\
\hline $\mathrm{R}$ & $5.31^{*}$ & $4.17^{*}$ & 2.73 & $4.28^{*}$ & $4.38^{*}$ & $13.34^{* * *}$ & $12.35^{* *}$ & 4.66 & $3.67^{*}$ & $5.18^{*}$ & $22.49^{* * *}$ & 3.12 \\
$\mathrm{G}$ & $5.75^{* *}$ & 3.17 & $4.19^{*}$ & $16.38^{* * *}$ & $4.28^{*}$ & $13.37^{* * *}$ & $13.93^{* *}$ & $18.06^{* * *}$ & $4.87^{*}$ & $6.90^{* *}$ & $34.62^{* * *}$ & $9.20^{* * *}$ \\
$\mathrm{~B}$ & 1.10 & 3.00 & 0.39 & 0.95 & 0.57 & $8.08^{* *}$ & $18.63^{* * *}$ & 1.80 & 0.13 & $9.38^{* * *}$ & $16.57^{* * *}$ & 3.67 \\
Leaf Length & 0.42 & 2.26 & 2.47 & 0.77 & 1.68 & 1.30 & 2.12 & 0.44 & 1.17 & 3.12 & 0.63 & 0.02 \\
Leaf Width & 0.94 & 0.48 & 1.51 & 1.56 & 3.41 & 3.35 & 1.61 & 0.55 & $3.68^{*}$ & 1.21 & 2.21 & 1.79 \\
Leaf Area & 0.60 & 1.22 & 1.93 & 1.51 & 2.22 & 3.33 & 1.87 & 0.21 & 2.65 & 1.17 & 1.99 & 1.28 \\
Leaf Perimeter & 0.28 & 2.40 & 2.56 & 0.80 & 0.60 & 1.94 & 2.43 & 0.40 & 0.34 & 2.51 & 1.55 & 0.14 \\
AL_ratio & 0.94 & 0.48 & 1.51 & 1.57 & 3.41 & 3.35 & 1.61 & 0.55 & $3.68^{*}$ & 1.21 & 2.21 & 1.78 \\
AP_ratio & 0.92 & 0.50 & 1.55 & 1.66 & 3.29 & $3.51^{* *}$ & 1.69 & 0.60 & $3.55^{*}$ & 1.28 & 2.28 & 1.56 \\
Eccentricity & 1.14 & 0.22 & 1.93 & 1.16 & $9.98^{* * *}$ & $6.12^{* *}$ & 3.34 & 0.46 & $6.05^{* *}$ & $5.08^{*}$ & $5.16^{*}$ & 0.44 \\
Rectangularity & 0.91 & 2.21 & 0.10 & 2.44 & 1.19 & 1.06 & 0.15 & 0.96 & 0.29 & 1.09 & 0.63 & 0.36 \\
Area Convexity & 0.91 & 2.21 & 0.10 & 2.44 & 1.19 & 1.06 & 0.15 & 0.79 & 0.29 & 1.09 & 0.63 & 0.24 \\
Circularity & 1.06 & 0.09 & 1.19 & 1.32 & $4.25^{*}$ & 3.03 & 1.20 & 0.54 & $4.91^{*}$ & 1.37 & 2.34 & 2.20 \\
Form Factor & 1.07 & 0.07 & 1.13 & 1.51 & $4.20^{*}$ & 3.39 & 1.27 & 0.74 & $4.79^{*}$ & 1.56 & 2.44 & 1.83 \\
\hline
\end{tabular}
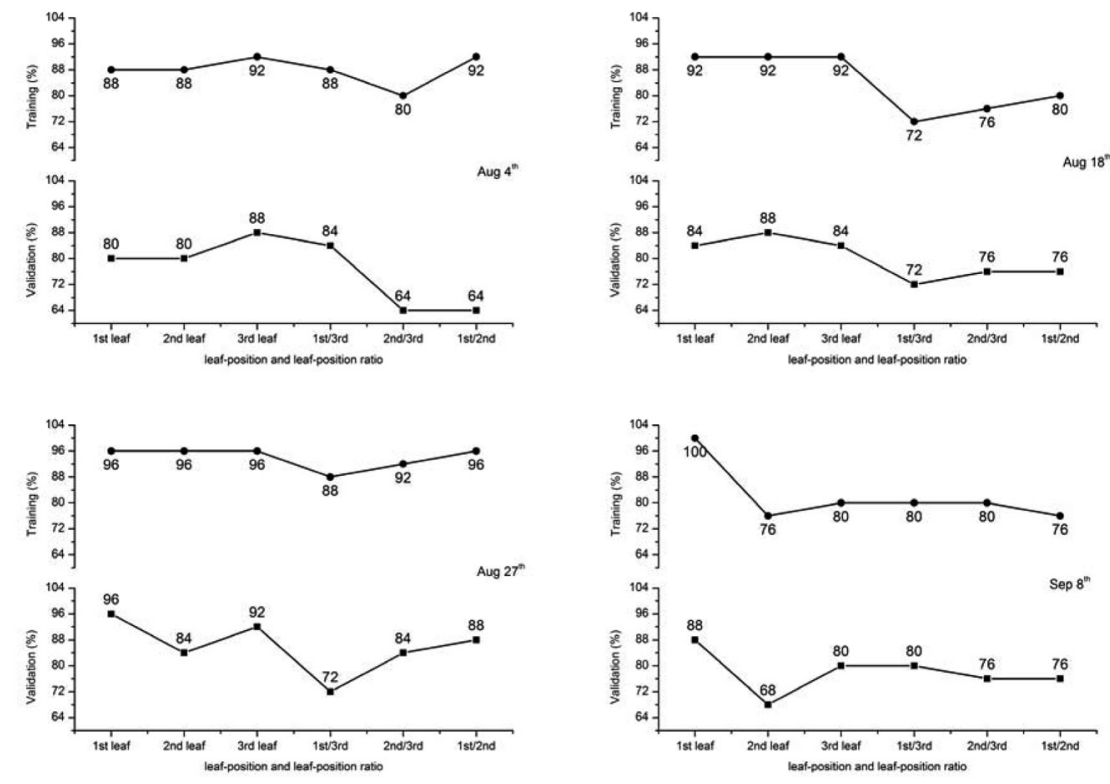

Figure 2. The SVM training accuracy and LOO-CV validation accuracy of the different leaf-positions in different growth stages 
Figure 2. indicates the optimized leaf-position (leafposition ratio) whose characteristic parameters can be used to identification rice nitrogen stress with the maximum accuracy. But in order to know the identifying accuracy of different degrees of nitrogen stress, paper had done further research. On August 4th, the research finds the identification accuracy were up to $100 \%$ under $\mathrm{N} 1$ and $93 \%$ under N2 (the left misdiagnosed to be N3). But, under $\mathrm{N} 3$ the identification misdiagnosed to be medium nitrogen deficiency was $80 \%$. On August $18^{\text {th }}$, recognition rate all achieved $100 \%$ under N1 and N2; under N3 it was $60 \%$ with the left misdiagnosed to be N2. On August $27^{\text {th }}$, under N1 the identification accuracy was $80 \%$ with left misdiagnosed to be N2; under $\mathrm{N} 2$ and N3, they were up to $100 \%$.

\subsection{Identification results with fisher discriminate analysis fisher}

After optimized choice of leaf samples' characteristic parameters by one-way ANOVA, chose Sig. value less than 0.05 from different leaf-position to diagnose rice's nitrogen stress by Fisher discriminate analysis. On August $4^{\text {th }}, 18^{\text {th }}, 27^{\text {th }}$ and September $8^{\text {th }}$, for different degree of nitrogen stress the maximum training accuracy respectively were $92 \%$ ( $3^{\text {rd }}$ leaf $), 92 \%\left(1^{\text {st }}\right.$ and $2^{\text {nd }}$ leaf $), 100 \%$ ( $1^{\text {st }}$ leaf $)$, and $96 \%$ ( $1^{\text {st }}$ leaf $)$, and the corresponding validation accuracy respectively were $80 \%, 92 \%, 96 \%, 88 \%$. On August $18^{\text {th }}$, with the $3^{\text {rd }}$ leaf's characteristic parameters the training accuracy was maximum for identifying too, but the model was not stable as the low validation accuracy $(88 \%)$. At the same growth period, to identify with the $1^{\text {st }}$ and $2^{\text {nd }}$ leaf's characteristic parameters resulted in the same training accuracy $(92 \%)$, but their validation accuracy increased to $92.00 \%$. So we didn't choose the $3^{\text {rd }}$ leaf on August $18^{\text {th }}$ (Figure 3).

After the identifying of rice nitrogen stress of every growth period by Fisher discriminate analysis, the study found the optimal leaf-position which had the maximum identifying accuracy of nitrogen stress. Table 4 shows the optimal leaf-position with the maximum identifying accuracy of nitrogen stress in every growth period by Fisher discriminate analysis. It was coincident with what tested by SVM. In this case, it means when diagnosing rice nitrogen stress by SVM the leaf-position with the maximum identifying accuracy is also the one which has the most obvious difference of physiological characteristic from different degree of nitrogen stress.

When identifying different degree of rice nitrogen stress, it was found that in different growth period the identification accuracy were different by using different leaf-position's characteristic parameters. With the $3^{\text {rd }}, 2^{\text {nd }}, 1^{\text {st }}$, and 1 st leaf's characteristic parameters from 4 growth period, respectively, Table 4 showed the maximum identifying accuracy by SVM and Fisher discriminant analysis. It agrees with the rice physiological characters under nitrogen stress in all growth period. On August $4^{\text {th }}$, rice was in the early part of tillering stage. Because of the mobility of nitrogen in rice plant, nitrogen deficiency symptom is mainly appeared in the 3rd leaf. And, at this period, rice requires little nitrogen for growth, so there's no significant difference between N2 and N3. On August $18^{\text {th }}$, most of nitrogen in the $3 \mathrm{rd}$ leaf had moved to the $1^{\text {st }}$ leaf, and nitrogen in the $2^{\text {nd }}$ leaf also gradually moved to the $1^{\text {st }}$ leaf. So the $2^{\text {nd }}$ leaf had the most significant difference of nitrogen deficiency symptom. The nitrogen content of the $2^{\text {nd }}$ leaf gradually stopped changing with time going, on August $27^{\text {th }}$ the $2^{\text {nd }}$ leaf almost stopped moving nitrogen to the $1^{\text {st }}$ leaf. At this time the 1 st leaf was affected by nitrogen deficiency, rice leaves showed the obvious nitrogen deficiency symptom. On September $8^{\text {th }}$, as a result of long-time nitrogen stress, only the $1^{\text {st }}$ leaf can show the difference of nitrogen deficiency symptom and the identifying accuracy can get the maximum.

Table 5 showed the rice leaves' characteristic parameters which were used to identify different degrees of nitrogen stress with the highest accuracy in 4 growth periods. That characteristic parameter mainly were spectral feature (R, G, and B band), leaf length, leaf width, leaf area, leaf perimeter, $\mathrm{AL}$ ratio, and AP_ratio, which agree with physiological characteristics of rice nitrogen deficiency. 

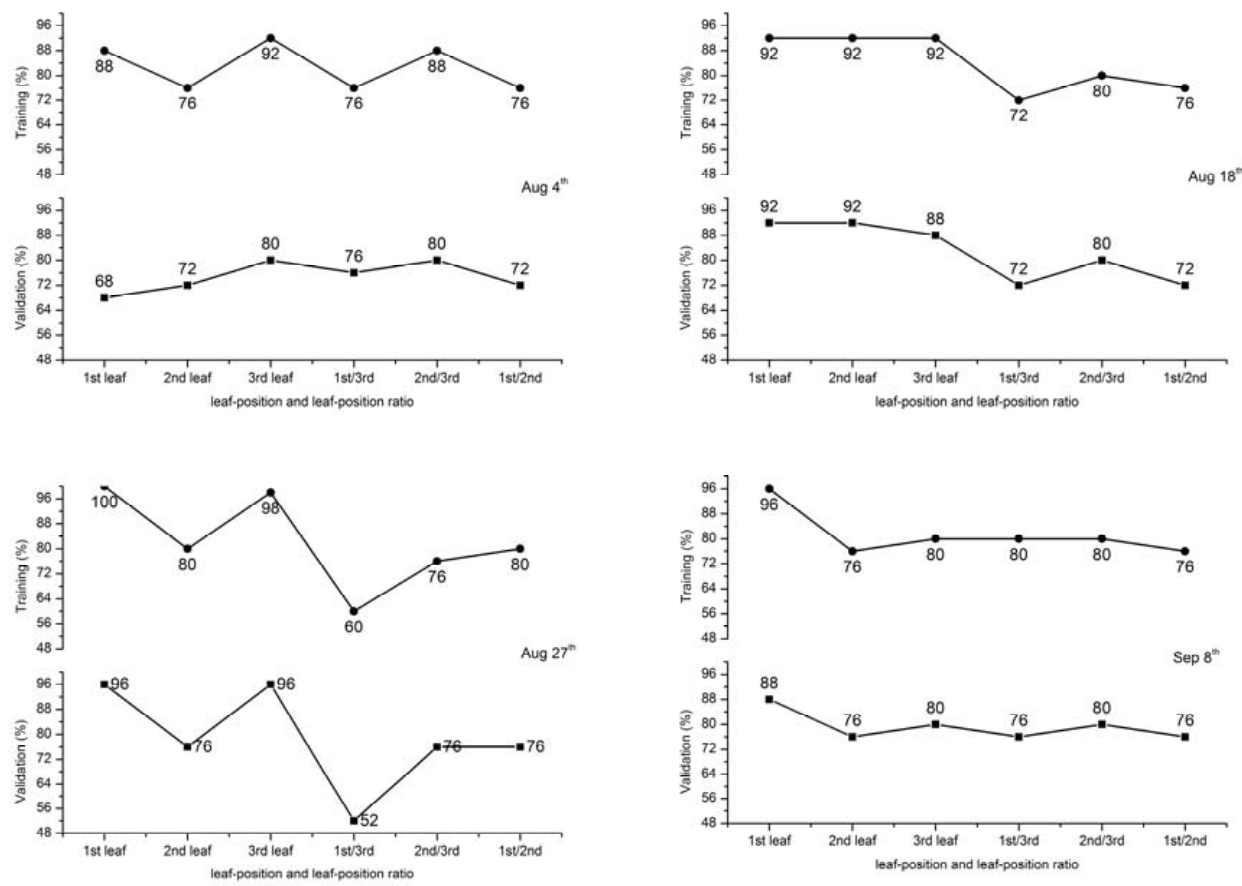

Figure 3. The Fisher discriminate analysis training accuracy and the validation accuracy of the different leafpositions at the different growth stages

Table 4. Comparison of classification accuracy with two classification methods for Different growth stages

\begin{tabular}{ccccc}
\hline Leaf-position (Growth period) & $3^{\text {rd }}$ leaf $(804)$ & $2^{\text {nd }}$ leaf $(818)$ & $1^{\text {st }}(827)$ & $1^{\text {st }}$ leaf $(908)$ \\
\hline SVM & $92 \%$ & $92 \%$ & $96 \%$ & $100 \%$ \\
LOO-CV & $88 \%$ & $88 \%$ & $96 \%$ & $88 \%$ \\
\hline Discriminate Analysis & $92 \%$ & $92 \%$ & $100 \%$ & $96 \%$ \\
LOO-CV $^{\text {a }}$ & $80 \%$ & $92 \%$ & $96 \%$ & $88 \%$ \\
\hline
\end{tabular}

a. LOO-CV only conducted in the cases in this analysis. In validation, every case is sorted by the function derived by all cases but this one

By analyzing characteristic parameters which could be used to identify the nitrogen with the highest accuracy in 4 growths periods, the universal characteristic parameters were selected to identify the nitrogen stress of rice for all growth period.
Finally, R, G, Leaf Length, Leaf Area, and Leaf Perimeter were used as 5 universal characteristic parameters of all growth period to diagnose the nitrogen stress by SVM. 
Table 5. Rice leaves' characteristic parameters were selected to diagnose the nitrogen stress with the highest accuracy

\begin{tabular}{ccccc}
\hline Leaf-position (Growth period) & $3^{\text {rd }}$ leaf $(804)$ & $2^{\text {nd }}$ leaf $(818)$ & $1^{\text {st }}(827)$ & $1^{\text {st }}$ leaf $(908)$ \\
\hline R & $\checkmark$ & $\checkmark$ & $\checkmark$ & \\
G & $\checkmark$ & $\checkmark$ & $\checkmark$ & $\checkmark$ \\
B & $\checkmark$ & $\checkmark$ & $\checkmark$ & \\
Leaf Length & $\checkmark$ & $\checkmark$ & $\checkmark$ & $\checkmark$ \\
Leaf Width & $\checkmark$ & $\checkmark$ & $\checkmark$ & \\
Leaf Area & $\checkmark$ & $\checkmark$ & $\checkmark$ & $\checkmark$ \\
Leaf Perimeter & $\checkmark$ & $\checkmark$ & $\checkmark$ & $\checkmark$ \\
AL_ratio & $\checkmark$ & $\checkmark$ & $\checkmark$ & \\
AP_ratio & $\checkmark$ & $\checkmark$ & $\checkmark$ & \\
Eccentricity & & $\checkmark$ & $\checkmark$ & \\
Rectangularity & & $\checkmark$ & $\checkmark$ \\
Area Convexity & & $\checkmark$ & $\checkmark$ \\
Circularity & & $\checkmark$ & $\checkmark$ & \\
Form Factor & & $\checkmark$ & $\checkmark$ & \\
\hline
\end{tabular}

Table 6. The identifying accuracy of nitrogen stress using the universal character parameters for different growth stages

\begin{tabular}{ccccc}
\hline Leaf-position (Growth period) & $3^{\text {rd }}$ leaf $(804)$ & $2^{\text {nd }}$ leaf $(818)$ & $1^{\text {st }}(827)$ & $1^{\text {st }}$ leaf $(908)$ \\
\hline SVM & $92 \%$ & $92 \%$ & $100 \%$ & $96 \%$ \\
LOO-CV $^{\text {a }}$ & $80 \%$ & $92 \%$ & $100 \%$ & $84 \%$ \\
\hline
\end{tabular}

a. LOO-CV only conducted in the cases in this analysis. In validation, every case is sorted by the function derived by all cases but this one

Results shown in Table 6, using the universal character parameters to identify the nitrogen stress of rice could get the high training accuracy $(92 \%, 92 \%$, $100 \%$ and $96 \%)$, and validation accuracy $(80 \%, 92 \%$, $100 \%$ and $84 \%$ ) for 4 growth periods.

\section{Conclusions}

This paper takes the three most anterior leaves of rice under different nitrogen nutrition condition as the object of research. In laboratory condition, spectral and morphological characteristic parameters were 
acquired from the scanning image of rice leaves to diagnose the nitrogen status. Also this study introduce characteristic parameter ratio to increase identification accuracy. Finally, SVM is used to identify the rice's nitrogen nutrient level.

During four growth periods, with universal characteristic parameter the overall recognition rates of different nitrogen nutrition level are 92\%, $92 \%, 100 \%$ and $96 \%$, respectively. In detail, N1's recognition rates are the highest, which all reach $100 \%$. And N2's recognition rates are $93 \%, 100 \%$, $100 \%$, and $93 \%$, respectively, and N3's recognition rates are $80 \%, 60 \%, 100 \%$, and $100 \%$, respectively. On August $4^{\text {th }}, 20 \%$ of N3 samples are misclassified as N2, On August 18th, 40\% of N3 samples are misclassified as N2, so it may be difficult to identify $\mathrm{N}$ deficiency in very early growth period. And in other growth periods, the recognition rates are high for different $\mathrm{N}$ nutrient status.

The study provides evidence for quick diagnosis of $\mathrm{N}$ nutrient status, which makes it possible to accurately identify rice nitrogen stress with scanning technology and SVM.

With a portable scanner, the images of rice leaves can be collected quickly by the static scanning technology under field conditions, and the rice nitrogen stress might be identified for guiding fertilization in time. Other crops such as maize, wheat with nitrogen stress, they usually have some symptom by the shape and color of leaf, so this method can be used to diagnose the nitrogen situation for them. So, the technology introduced in the study has the application value and development potential.

\section{Acknowledgements}

The author thanks Mr. Cai guangzhe for helping with scanning rice leaves. The author also thanks the Professor Wang Ke for his constant encouragement for the work. Financial assistance is from the National Natural Science Foundation of China (Grant No.31172023)

\section{References}

Bacci, L., De Vincenzi, M., Rapi, B. 1998. Two Methods for The Analysis of Colorimetric Components Applied to Plant Stress Monitoring. Comput. Electron. Agr. 19, 167-186.

Brown, L., Jin, M. C., Leblanc, S.G. 2000. A Shortwave Infrared Modification to The Simple Ratio for LAI Retrieval in Boreal Forests: An Image and Model Analysis. Remote. Sens. Environ. 71, 16-25.

Burges Christopher, J.C. 1998. A Tutorial on Support Vector Machines for Pattern Recognition. Data. Min. Knowl. 2, 121-167.

Chen, J., Chen, X., Li, X. 2008. Study on Discrimination of Corn Seed Based on NearInfrared Spectra and Artificial Neural Network Model. Spectrosc. Spect. Anal. 28(8), 1806-1809.

Deng, N.Y., Tian., Y.J. 2004. New Method of Data Minning-Support Vector Machine. Science Press.

Deng, N.Y., Tian, Y.J. 2004. New Method of Data Minning-Support Vector Machine. Science Press.

Guan, Z.X., Tang, J., Yang, B.J. 2010. Study on Recognition Method of Rice Disease Based on Image. Chin. J. Rice Sci. 24(5), 497-502.

Jia, L.L., Fan, M.S., Zhang, F.S. 2009. Nitrogen Status Diagnosis of Rice by Using a Digital Camera. Spectrosc. Spect. Anal. 29(8), 2176-2179.

Jiang, L.G., Cao, W.X., Jiang, D. 2004. Distribution of Leaf Nitrogen, Amino Acids and Chlorophyll in Leaves of Different Positions and Relationship with Nitrogen Nutrition Diagnosis in Rice. Acta. Agronomica. Sinica. 30(8), 739-744.

Li, L.M., Guo, R.F., Guo, Z.J. 2007. Studies on Differences in Nitrogen Nutrition of Rice Genotypes. Journal of Northwest A \& F University (Nat. Sci. Ed.). 35(11), 75-79. 
Li, G.Z., Wang, M., Zen, H. J. 2004. An Introduction to Support Vector Machines and Other Kernel Based Learning Methods. Bejing: Publishing House of Electronics Industry Press.

Lu, J.W., Li, R. 2010. Common Rice Nutrient Deficiency Symptom and Correction Technology. Chinese Agricultural Press.

Milos, K., Branislav, B., Bosko, G. 2010. Soil Type Classification and Estimation of Soil Properties Using Support Vector Machines. Geoderma. 154, 340-347.

Nemmour, H.Y., Chibani, Y. 2006. Multiple Support Vector Machines for Land Cover Change Detection: An Application for Mapping Urban Extensions. ISPRS J. Photogramm. 61(2), 125133.

Shi, J.J., Liu, Z.Y., Zhang, L.L. 2009. Hyperspectral Recognition of Rice Damaged by Rice Leaf Roller Based on Support Vector Machine. Chin. J. Rice Sci. 23(3), 331-334.

Tracy, M., Blackmer, J., Schepers, S. 1996. Aerial Photography to Detect Nitrogen Stress in Corn. J. Plant Physiol. (148), 440-444.
Wang, B. 2011. Application of Clustering-Based Decision Tree in the Screening of Maize Germplasm. Agr. Sci. Tech-Hunan. 12(10), 14491452.

Wang, B. 2011. Application of Clustering-Based Decision Tree in the Screening of Maize Germplasm. Agr. Sci. Tech-Hunan. 12, 1449-1452.

Wei, H., Yoshiteru, N., Wang, S.Y. 2005. Forecasting Stock Market Movement Direction with Support Vector Machine. Comput. Oper. Res. 32, 25132522 .

Wu, L., Luo, H.Z., Zhu, M.H. 2011. Efficient LeaveOne-Out Evaluation of SVM Models. Proceedings of 2011 IEEE International Conference on Intelligent Computing and Intelligent Systems Vol. 3.

Zhao, Q.L., Wang, K.R., Ma, J.Q. 2009. Effects of Long-Term Application of Different Fertilizer Patterns on Rice Paddy Soil Nitrogen and Rice Nitrogen Nutrition. Acta. Agronomica. Sinic. 35(8), 1539-1545.

Zhu, J.X., Deng, J.S., Shi, Y.Y 2009. Diagnosis of Rice Nitrogen Status Basedon Characteristics of Scanning Leaf. Spectrosc. Spect. Anal. 29(8), 2171-2175. 